\title{
A Fine-grained Localization Scheme Using A Mobile Beacon Node for Wireless Sensor Networks
}

\author{
Kezhong Liu* and Ji Xiong*
}

\begin{abstract}
In this paper, we present a fine-grained localization algorithm for wireless sensor networks using a mobile beacon node. The algorithm is based on distance measurement using RSSI. The beacon node is equipped with a GPS sender and RF (radio frequency) transmitter. Each stationary sensor node is equipped with a RF. The beacon node periodically broadcasts its location information, and stationary sensor nodes perceive their positions as beacon points. A sensor node's location is computed by measuring the distance to the beacon point using RSSI. Our proposed localization scheme is evaluated using OPNET 8.1 and compared with Ssu's and Yu's localization schemes. The results show that our localization scheme outperforms the other two schemes in terms of energy efficiency (overhead) and accuracy.
\end{abstract}

Keywords—Localization Algorithm, Mobile Beacon Node, Sensor Network, RSSI

\section{INTRODUCTION}

Wireless sensor networks (WSNs) are expected to be used in a wide range of applications, such as target tracking, military reconnaissance, habitat sensing, and fire detection $[1,2]$. In most applications, sensed data without knowing the sensors' location is meaningless. Localization must be considered as an implicit feature of a sensor network. This can significantly simplify network discovery and maintenance efforts and lead to substantial energy savings. The localization problem has received much attention in recent literature. Most of the current localization schemes are proposed to eliminate a GPS receiver on every sensor node. They suggest the use of a special type of nodes, "beacon nodes", which know their absolute locations via GPS or manual placement. Other sensors can discover their locations based on information provided by these anchor nodes [3].

Several schemes, broadly classified into two categories range-based and range-free schemes, have been proposed to deal with the localization problem. Firstly, the range-based schemes need either node-to-node distances or the angles to estimate locations [5-8]. The information can be obtained using time of arrival (TOA), time difference of arrival (TDOA), angle of arrival (AOA), and the received signal strength indicator (RSSI) technologies $[9,10]$. The range-based schemes typically have higher location accuracy but require additional hardware to measure the distances or angles. Secondly, several approaches based on range-free are proposed to determine senor

※ Supported by the National Natural Science Foundation of China under Grant No.60703099, the China Postdoctoral Science Foundation under Grant No. 20080440969.

Manuscript received February 5, 2010; accepted May 28, 2010.

Corresponding Author: Ji Xiong

* Department of Traffic Information Engineering and Control, Wuhan University of Technology; Wuhan, China(bearxiongji@163.com) 
node locations in order to reduce the localization cost in wireless sensor networks. A simple approach proposed in this paper computes centroid locations of beacon nodes, which approximate sensor node locations. For fitting in with the distinguishing characteristics of wireless sensor networks, some approaches estimate the location without distance or angle information. Belusu and Heidemann [11] introduced Centroid formula to calculate the nodes' locations based on receive beacons containing reference points' positions. Although the range-free schemes do not need distance or angle information for localization, the range-free schemes cannot accomplish high precision as the range-based schemes. Our focus is on the fine-grained localization scheme that outperforms the Ssu and Yu's location estimation methods.

At the same time, some scholars have also put forward a number of mobility models. We can take advantage of these models to build a variety of location methods, such as Random Walk, Random Waypoint, Random Direction, Gauss-Markov.

1. Random Walk Mobility Model (including its many derivatives): A simple mobility model based on random directions and speeds.

2. Random Waypoint Mobility Model: A model that includes pause times between changes in destination and speed.

3. Random Direction Mobility Model: A model that forces MNs to travel to the edge of the simulation area before changing direction and speed.

4. Gauss-Markov Mobility Model: A model that uses one tuning parameter to vary the degree of randomness in the mobility pattern.

These mobility models have their respective advantages and disadvantages. By summing up the advantages and disadvantages of these models. We can choose the most suitable mobility model in support of our algorithms.

Random Walk Mobility Model: a MN(mobile beacon node) moves from its current location to a new location by randomly choosing the direction and speed in which to travel. The new speed and direction are both chosen from predefined ranges, [minspeed, maxspeed] and [0;2ח] respectively. Each movement in the Random Walk Mobility Model occurs in either a constant time interval $t$ or a constant distance traveled $d$, at the end of which a new direction and speed are calculated. If a MN which moves according to this model reaches a simulation boundary, it "bounces" off the simulation border with an angle determined by the input direction. The MN then continues along this new path.

The Random Walk Mobility Model is a memoryless mobility pattern because it retains no knowledge concerning its past locations and speed values[12]. The current speed and direction of a $\mathrm{MN}$ is independent of its past speed and direction[13].

Nevertheless, the Random Waypoint Mobility Model includes the pause times between changes in direction and/or speed [14]. A MN begins by staying at one location for a certain period of time (i.e., a pause time). Once this time expires, the $\mathrm{MN}$ chooses a random destination in the simulation area and a speed that is uniformly distributed between [minspeed, maxspeed]. The MN then travels toward the newly chosen destination at the selected speed.

In most of the performance investigations that use the Random Waypoint Mobility Model, the MNs are initially distributed randomly around the simulation area. This initial random distribution of MNs is not representative of the manner in which nodes distribute themselves when moving. 


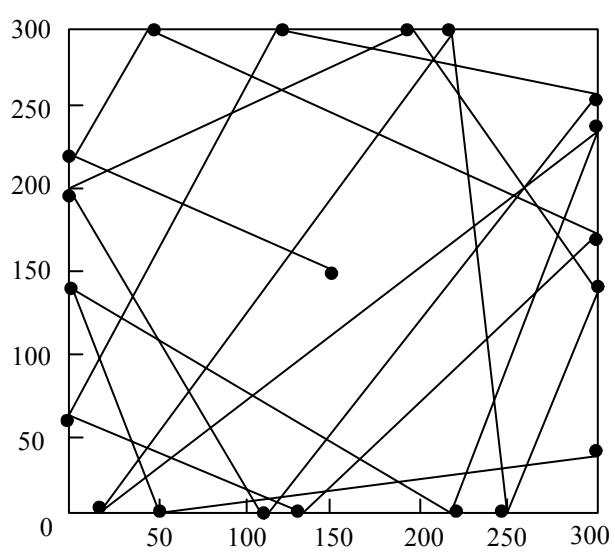

Fig. 1. Traveling pattern of an MN using the Random Direction Mobility Model

The Random Direction Mobility Model [15] was created to overcome the density waves in the average number of neighbors produced by the Random Waypoint Mobility Model. A density wave is the clustering of nodes in one part of the simulation area.

In order to alleviate this type of behavior and promote a semi-constant number of neighbors throughout the simulation, the Random Direction Mobility Model was developed [15]. In this model, MNs choose a random direction in which to travel similar to the Random Walk Mobility Model. A MN then travels to the border of the simulation area in that direction. Once the simulation boundary is reached, the MN pauses for a specified time, chooses another angular direction (between 0 and 180 degrees) and continues the process.

Figure. 1 shows an example path of a $\mathrm{MN}$, which begins in the center of the simulation area or position $(150,150)$, using the Random Direction Mobility Model.

The Gauss-Markov Mobility Model was designed to adapt to different levels of randomness via one tuning parameter. Initially each $\mathrm{MN}$ is assigned a current speed and direction. At fixed intervals of time, $n$, movement occurs by updating the speed and direction of each MN. The following equations are the Gauss-Markov Mobility Model.

You can assume that $(x n, y n)$ and $(x n-1, y n-1)$ are the $x$ and $y$ coordinates of the MN's position at the $n^{\text {th }}$ and $(n-1)^{\text {th }}$ time intervals, respectively. A random variable using the following equations:

$$
\begin{aligned}
& S n=\alpha S n-1+(1-\alpha) \bar{S}+\sqrt{\left(1-\alpha^{2}\right)} S_{X_{n-1}} \\
& d n=\alpha d n-1+(1-\alpha) \bar{d}+\sqrt{\left(1-\alpha^{2}\right)} d_{X_{n-1}}
\end{aligned}
$$

The MN's position is given by the following equations:

$$
\begin{aligned}
& X_{n}=X_{n-1}+S_{n-1} \cos d_{n-1} \\
& y_{n}=y_{n-1}+S_{n-1} \sin d_{n-1}
\end{aligned}
$$




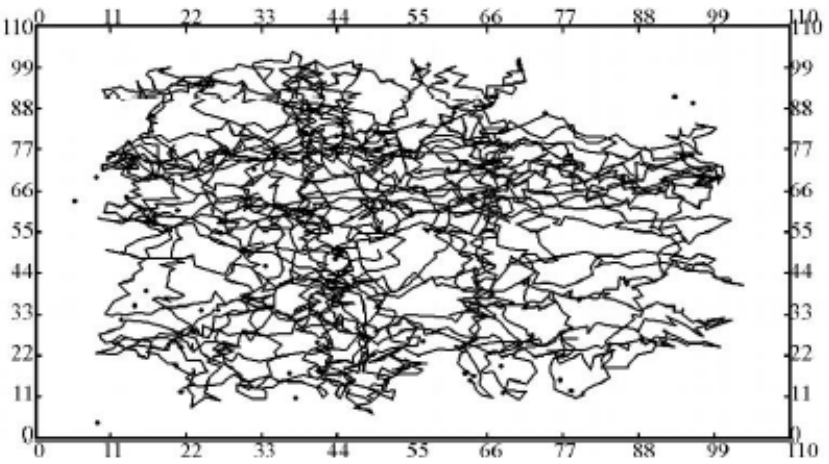

Fig. 2. Traveling pattern of an MN using the Gauss-Markov Mobility Model

$S_{n}$ and $d n$ are representing $n$ times value of speed and direction.

$S$ and $d$ are constants representing the mean value of speed and direction.

$S_{X_{n-1}}$ and $d_{X_{n-1}}$ are random variables from a Gaussian distribution.

$\alpha$. is intermediate levels of randomness, the value of is between 0 and 1 .

Figure 2 illustrates an example traveling pattern of a MN using the Gauss-Markov Mobility Model. The MN begins its movement in the center of the simulation area or position $(55,55)$ and moves for 2500 seconds.

In Figure 2, with a broadcast information interval of $1 \mathrm{~s}, \alpha$ is 0.6 , and $S_{X_{n-1}}$ and $d_{x_{n-1}}$ are chosen from a random Gaussian distribution with mean equal to zero and standard deviation equal to one. The value of $s$ is fixed at $2 \mathrm{~m} / \mathrm{s}$; the value of $\mathrm{d}$ is initially 90 degrees, but changes over time according to the edge proximity of the node[16].

By comparing the advantages and disadvantages of each model, we finally chose the Random Direction Mobility Model. As our sensor nodes are randomly distributed to the entire sensor area. If we want to locate all sensor nodes on the sensing area, we must make a MN traverse the entire sensing area and Random Direction Mobility Model move in straight line. Thus, the Random Direction Mobility Model is the best option.

In this paper, we propose a localization scheme based on geometric constraints as an extension of Ssu's scheme. In addition, we describe how to appropriately select two beacon points to achieve the smallest location error through computing angle. Section II overviews existing localization algorithms using a mobile beacon node with combination of Random Direction Mobility Model. In Section III, our proposed localization scheme is introduced. The simulation and discussion of the new localization scheme are presented in Section IV. Section V closes with a brief conclusion.

\section{RELATED WORK}

Recently, in some researches the sensor node locations by the use of mobile beacon node. Zhang Baoli [1], Yu [2] and Ssu [4] proposed a localization algorithm using a mobile beacon node. Sichitiu and Ramadurai [17] put forward a localization mechanism using a single mobile beacon transmitting its current location. Guo [18] proposed probabilistic localization schemes with a mobile beacon. In their algorithm, it is assumed that a mobile beacon node moves around 
in a sensor area and periodically broadcasts beacon messages that include its current location information.

In Ssu's algorithm, a stationary sensor node receives and records the first and the last mobile beacon node's locations when it moves within the communication range of the stationary sensor node. The first and last mobile beacon node's locations are the positions that mobile beacon node just enters and leaves the reception range of the sensor node, respectively. Ssu's scheme localizes sensor nodes without any distance or angle information, contrary to previous schemes, yet can estimate the location of sensor nodes as accurately as those schemes with distance or angle information.

In Ssu's algorithm, the reception range is defined as a boundary. On one side of the boundary, the sensor node can't receive any signal from the beacon nodes. While on the other side, the sensor node can receive signals without any errors from the beacon node. In reality, there is no such well defined boundary because a sensor node always has a certain error probability to receive a packet from a mobile beacon node. Beacon points in Ssu's algorithm are usually not on the same circle because the sensor node has a certain error probability for receiving a packet. To solve the problem, we take the maximum RSSI location as the beacon point where the sensor node has the smallest error probability in receiving a packet from a mobile beacon node [2]. The detail of our scheme will be given in Section III

Considering wireless communication channels, Yu proposes[2] a localization algorithm using a mobile beacon node. In his algorithm, a sensor node has a certain probability of receiving a packet from a mobile beacon node in a noisy environment. The probability of receiving a packet correctly becomes greater with the increasing SNR (signal to noise ratio). In his algorithm, maximum RSSI locations are recorded as beacon points, where the sensor node has the smallest error probability in receiving a packet from the mobile beacon node, as shown in Fig.3. After the sensor node obtains the maximum RSSI points B1 and B2, lines L01 and LO2 can be drawn perpendicular to lines A1B1 and A2B2, respectively. The intersection of L01 and LO2 is the sensor node location [2].

Although Yu's localization scheme performs constantly well and gives a higher accuracy than Ssu's algorithm in wireless communication environment, it is not energy-efficient. The sensor node continually listens to the mobile beacon node and records its locations when it is in the sensor node's reception range. However the only useful information is the maximum RSSI location and other received locations are eventually useless. To overcome the problem, we propose a

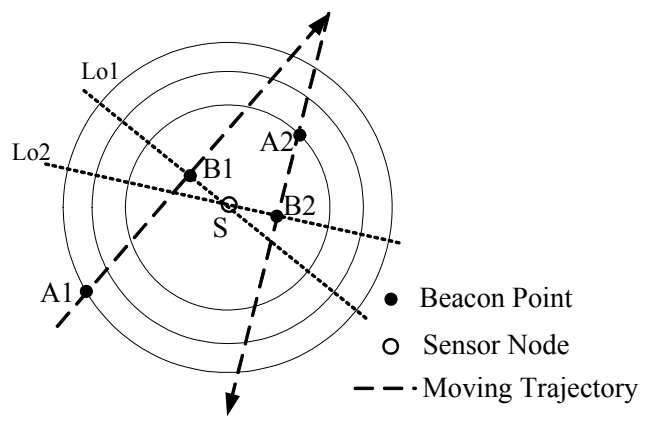

Fig. 3. Determination of sensor node location 
fine-grained localization scheme and a low-cost localization algorithm.

\section{Algorithm Design}

The basic concept of the proposed approach is that the stationary sensors can localize themselves by measuring the distance to a mobile beacon node when it comes into their reception range. Our localization algorithm can be divided into two steps.

Step 1. Localization by a mobile beacon node;

Step 2. Subsequent to step 1, there may be some sensor nodes which are not covered by the mobile beacon node. The localized sensor nodes can serve as stationary beacon nodes to localize its neighbors using the algorithms mentioned in Section III. In this section the procedures of the proposed localization scheme are discussed in detail. We also present a technique to reduce the energy consumption.

\subsection{Assumption}

The proposed localization scheme is based on the following assumptions:

1. Stationary sensor nodes are deployed in an ad hoc fashion and they are self-configured.

2. A mobile beacon node moves over the sensor field to broadcast its location information. The mobile beacon node is equipped with a GPS and RF transmitter. The stationary nodes are equipped with a RF transmitter and can receive mobile beacon power signals and messages.

3. The mobile node uses the Random Direction Mobility Model, which moves in a straight line.

4. The mobile node is not restrained by energy usage.

\subsection{Angle Calculation}

Beacon Point Selection: In this paper, a mobile beacon node periodically broadcasts beacon messages when it moves into the stationary sensor network. A beacon message contains the id, location, and timestamp information of the beacon point. Every sensor node maintains a visitor list. In Fig.4, Point $A$ is considered as the first approximate mobile beacon node's location when it moves within the communication range of the stationary sensor node, so $A$ point is the beacon point. At the point, the mobile beacon $M$ broadcasts a packet to the sensor node $B$. The packet has some important information, including current position, id and timestamp of the $A$ beacon point. Then the sensor $B$ receives the packet and it can compute the distance between $A$ and $B$ based on RSSI. The mobile beacon $M$ still moves into the sensor node's communication range. It periodically broadcasts localization messages, as shown in Fig. 5. When the mobile beacon $M$ moves to the point $T 4$, the sensor node $B$ finds that an energy signal from the mobile beacon node that is less than that of the $C$ point. So the sensor node $B$ obtains the maximum RSSI at point $C$ and the location is recorded as a beacon point. Line $B C$ can be drawn perpendicularly to line $A C$. Line $A C$ and $A B$ form an angle $\beta$. Line $A B$ and $A D$ form the other angle $a$. The pseudo-code of the beacon point's selection algorithm is presented as follows(Fig.4.). 


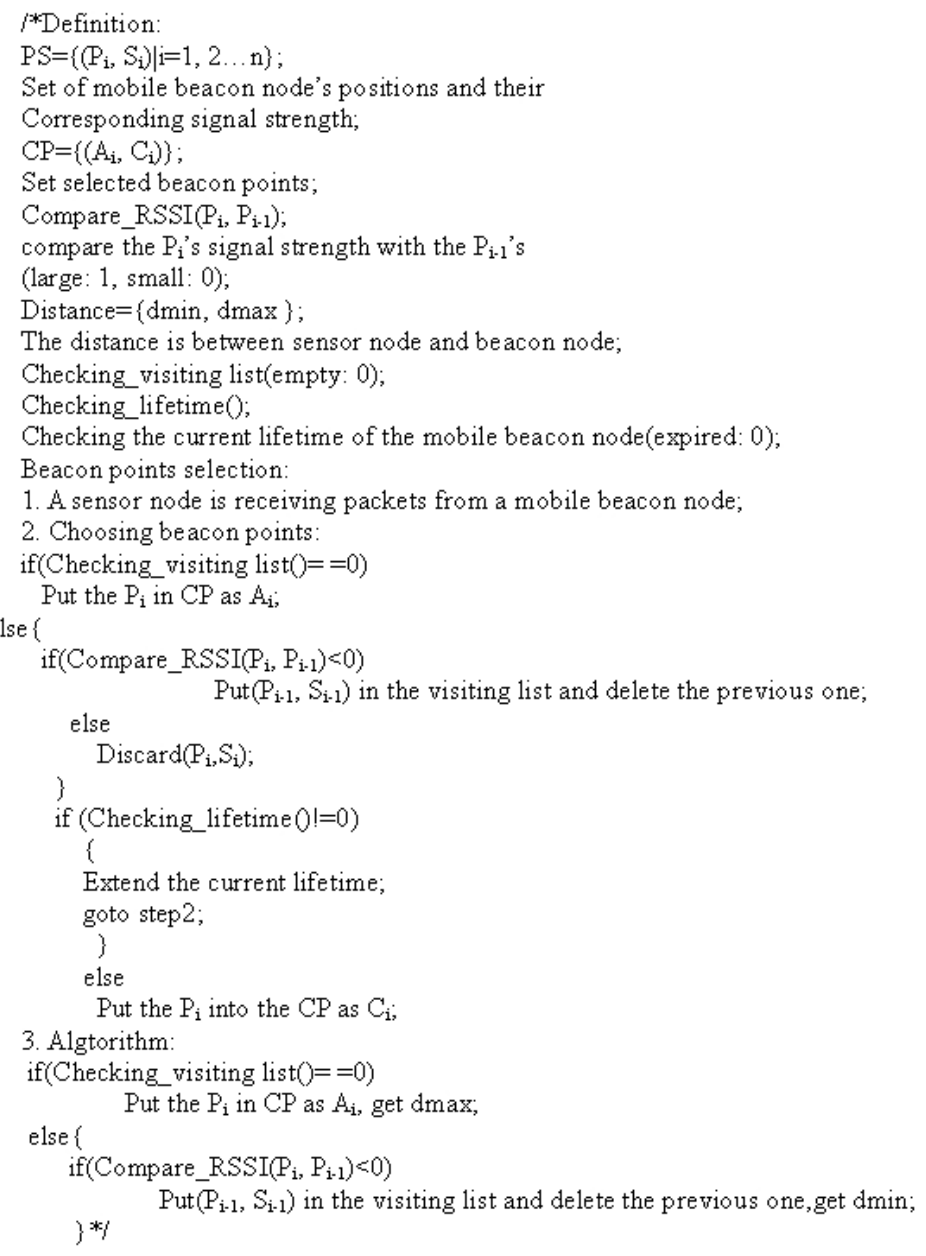

Fig. 4. The pseudo-code of beacon selection algorithm

\subsection{Localization Model}

After the stationary sensor node collects messages from two different beacon points, its location can be calculated (Fig.5.). The coordinates of the two positions, $A, C$ are $\left(x_{1}, y_{1}\right),\left(x_{2}, y_{2}\right)$ respectively. The stationary sensor node is $B$ point with the coordinate $\left(x_{0}, y_{0}\right)$. The communication radius of the mobile beacon and the stationary sensors are $R$. The distances between beacon points and the stationary node are $A B$ and $C B$ respectively. Hence we can obtain the following equations:

$$
\left\{\begin{array}{l}
\theta=|\arctan ((y 2-y 1) /(x 2-x 1))| \\
\beta=\arccos (A C / A B)
\end{array}\right.
$$




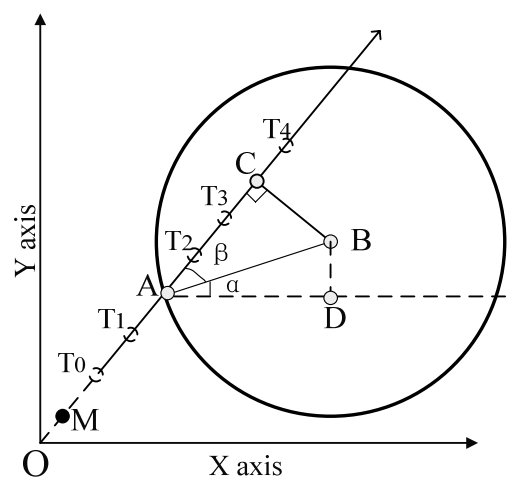

Fig. 5. Moving above stationary sensor node $B$

When we have $(y 2>y 1)$ and $(x 2>x 1)$, there are some mathematical models that can be defined. We can build a mathematical model one (Fig.5). :

$$
\begin{gathered}
\alpha=\theta-\beta \\
\left\{\begin{array}{l}
X_{0}=X_{1}+A B \cos \alpha \\
Y_{0}=Y_{1}+A B \sin \alpha
\end{array}\right.
\end{gathered}
$$

When mobile beacon node moves below the stationary sensor node, we can develop another set of mathematical models (Fig.6.).

$$
\begin{gathered}
\alpha=\theta+\beta \\
\left\{\begin{array}{l}
X_{0}=X_{1}+A B \cos \alpha \\
Y_{0}=Y_{1}+A B \sin \alpha
\end{array}\right.
\end{gathered}
$$

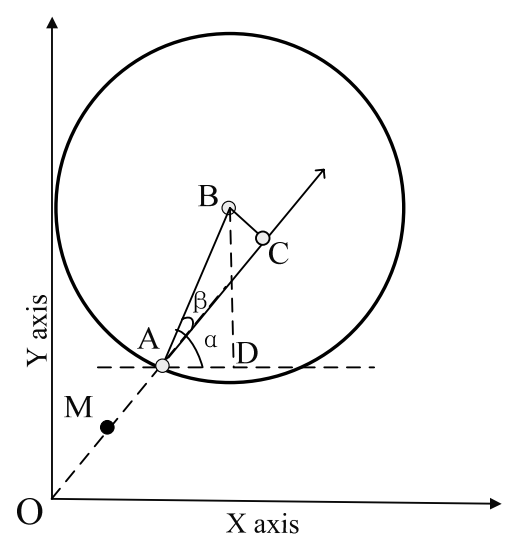

Fig. 6. Moving below stationary sensor node B 


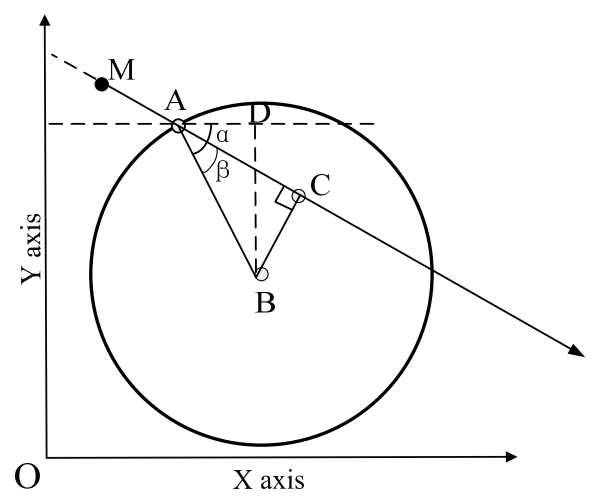

Fig. 7. Moving above stationary sensor node $B$

When we have $(y 2<y 1)$ and $(x 2>x 1)$, there are other mathematical models that can be defined. We can construct the following mathematical model two (Fig.7.).

$$
\begin{gathered}
\alpha=\theta+\beta \\
\left\{\begin{array}{l}
X_{0}=X_{1}+A B \cos \alpha \\
Y_{0}=Y_{1}-A B \sin \alpha
\end{array}\right.
\end{gathered}
$$

When the mobile beacon node moves below the stationary sensor node, we can develop another mathematical model (Fig.8.).

$$
\begin{gathered}
\alpha=\beta-\theta \\
\left\{\begin{array}{l}
X_{0}=X_{1}+A B \cos \alpha \\
Y_{0}=Y_{1}+A B \sin \alpha
\end{array}\right.
\end{gathered}
$$

When we have $\left(y_{2}<y 1\right)$ and $(x 2<x 1)$, there are other mathematical models that can be defined.

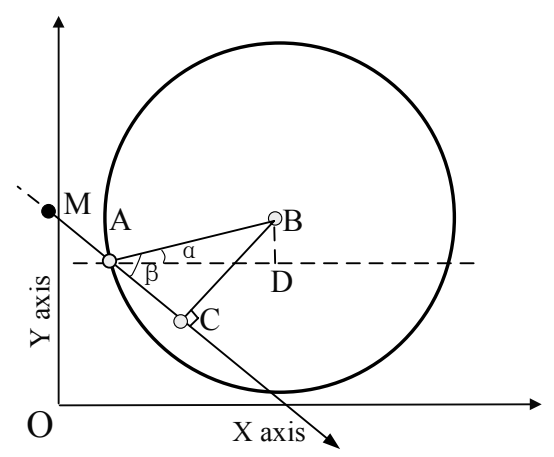

Fig. 8. Moving below stationary sensor node B 


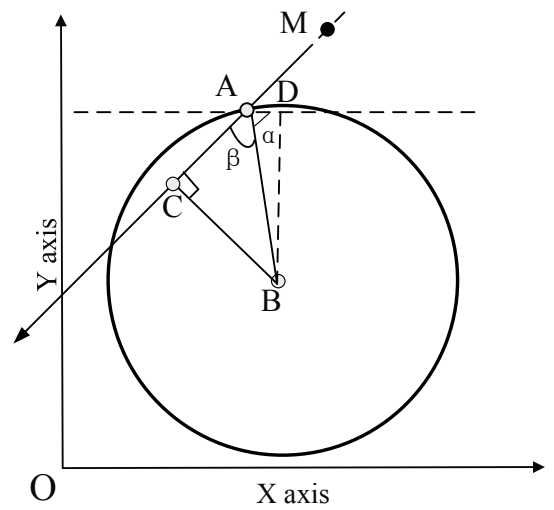

Fig. 9. Moving above stationary sensor node $B$

We can construct the following mathematical model three(Fig.9.).

$$
\begin{gathered}
\alpha=\pi-(\theta+\beta) \\
\left\{\begin{array}{l}
X_{0}=X_{1}+A B \cos \alpha \\
Y_{0}=Y_{1}-A B \sin \alpha
\end{array}\right.
\end{gathered}
$$

When the mobile beacon node moves below the stationary sensor node, we can develop another set of mathematical models (Fig.10.).

$$
\begin{gathered}
\alpha=(\beta-\theta) \\
\left\{\begin{array}{l}
X_{0}=X_{1}-A B \cos \alpha \\
Y_{0}=Y_{1}+A B \sin \alpha
\end{array}\right.
\end{gathered}
$$

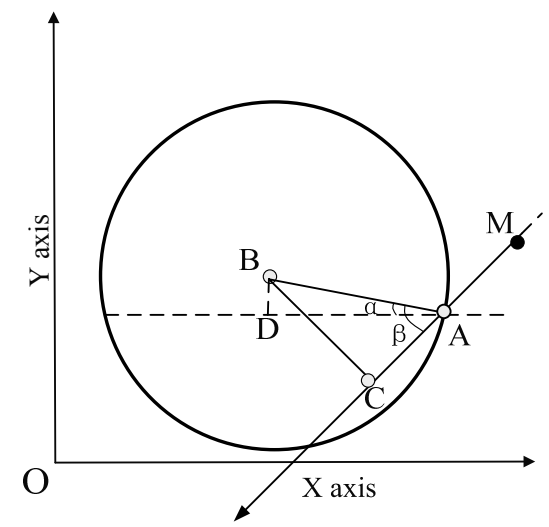

Fig. 10. Moving below stationary sensor node B 


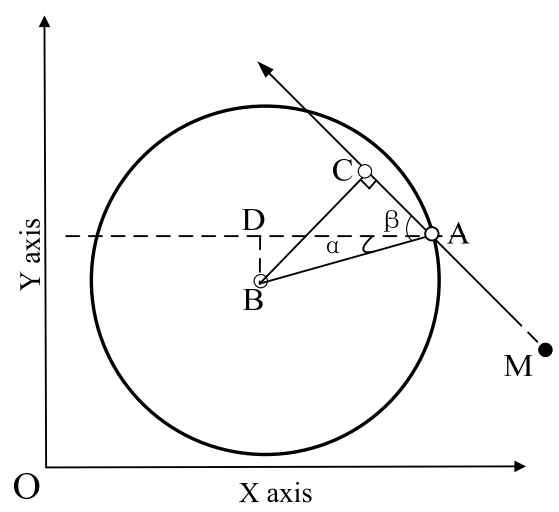

Fig. 11. Moving above stationary sensor node $B$

When we have $(y 2>y 1) a n d(x 2<x 1)$, there are other mathematical models that can be defined. We can construct the following mathematical model four (Fig.11.).

$$
\begin{gathered}
\alpha=\beta-\theta \\
\left\{\begin{array}{l}
X_{0}=X_{1}-A B \cos \alpha \\
Y_{0}=Y_{1}-A B \sin \alpha
\end{array}\right.
\end{gathered}
$$

When the mobile beacon node moves below the stationary sensor node, we can develop another set of mathematical models (Fig.12.).

$$
\begin{gathered}
\alpha=\pi-(\beta+\theta) \\
\left\{\begin{array}{l}
X_{0}=X_{1}+A B \cos \alpha \\
Y_{0}=Y_{1}+A B \sin \alpha
\end{array}\right.
\end{gathered}
$$

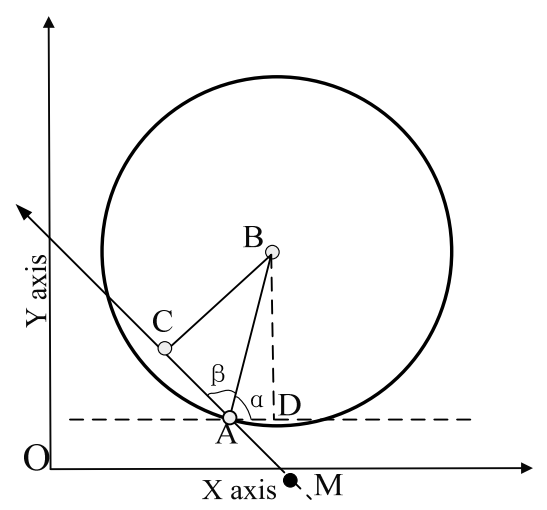

Fig. 12. Moving below stationary sensor node B 


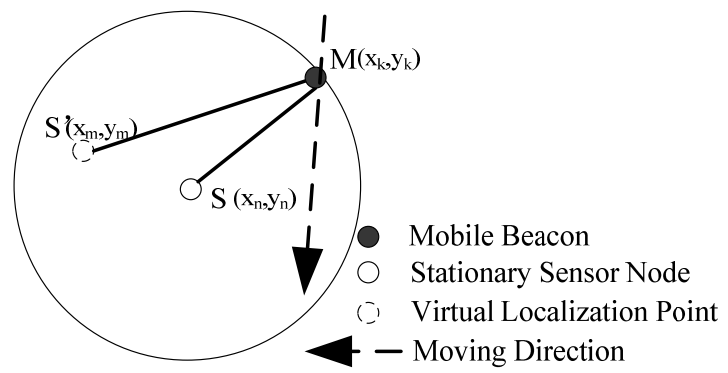

Fig. 13. Virtual point outside of the mobile beacon communication range

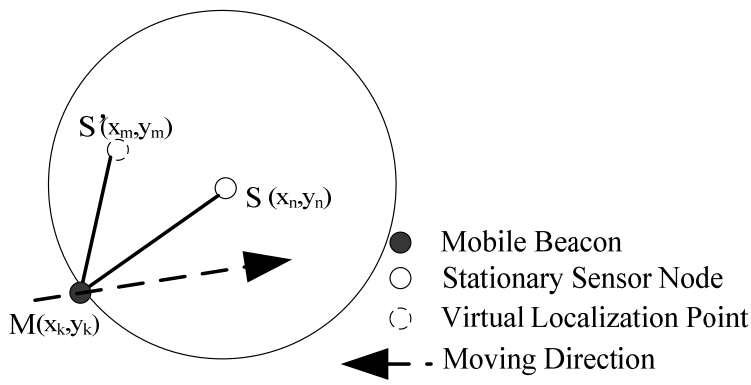

Fig. 14. Virtual point inside of the mobile beacon communication range

When the localization relationship between the mobile beacon node and the stationary node is consistent with one of the localization models described above, we can obtain two positions of the node according to the mathematical formula. However only one of them is the true coordinate of the node, so we need to choose from the two coordinates, as shown in Fig. 13 and Fig. 14.

We presume that node $\mathrm{A}$ has acquired its two positions $\left(x_{m}, y_{m}\right),\left(x_{n}, y_{n}\right)$ according to the model described above. When the mobile beacon moves to the location $\left(x_{k}, y_{k}\right)$ where beacon $A$ is within its communication range, the distances between the mobile beacon and the two positions can be calculated as follows:

$$
\left\{\begin{array}{l}
\left(x_{k}-x_{m}\right)^{2}+\left(y_{k}-y_{m}\right)^{2}=r_{m}^{2} \\
\left(x_{k}-x_{n}\right)^{2}+\left(y_{k}-y_{n}\right)^{2}=r_{n}^{2}
\end{array}\right.
$$

If $r_{m}<R$ and $r_{n}<R,\left(x_{m}, y_{m}\right)$ is the real localization of node $A$ and when $r_{m}<R$ and $r_{n}<R$, we select $\left(x_{m}, y_{m}\right)$ as the true position of node $A$. If $r_{m}<R$ and $r_{n}<R$ and $\left|r_{m}-R\right|<\left|r_{n}-R\right|$, we select $\left(x_{m}, y_{m}\right)$ as the true position of node $A$. If $r_{m}<R$ and $r_{n}<R$ and $\left|r_{m}-R\right|>\left|r_{n}-R\right|,\left(x_{m}, y_{m}\right)$ is real localization of node $A$

If a stationary sensor node does not collect locations of two beacon points, it may broadcast messages to its neighbors. Its localized neighbors in turn send back their locations. Based on the Newton iteration method [1], the sensor node can compute its location using its neighbors' locations. 


\section{SIMULATION}

In this section, we describe the simulation of our proposed localization algorithm in terms of overhead and location errors.

\subsection{Simulation Environment}

We used OPNET 8.1 to simulate our proposed algorithm. In simulations, the size of the sensor field is $150 * 150 \mathrm{~m} 2$, the communication radius of the mobile beacon and the stationary sensors are $R$, and 100 stationary sensor nodes were randomly deployed in the sensor area. The mobile beacon node was equipped with a GPS and RF transmitter. It moves in straight lines across the sensor area. Each stationary node was equipped with a RF. The beacon node has no energy restriction and each stationary sensor node is energy-restrained.

\subsection{Metrics}

Three metrics were employed to evaluate the performance of our localization algorithm.

1. Average overhead: It can be defined as [1] .

$$
P_{\text {avg }}=\frac{\sum_{i=1}^{N} P_{i}}{N}
$$

Where $P i$ denotes the overhead of stationary sensor node $i$. The average overhead for the three algorithms was simulated and results are shown in Fig.15. Since formula is relatively simple, the energy caused by the calculation can be neglected and compared with the energy consumption caused by other reasons[19].

We can clearly see that our localization scheme outperforms the other two schemes. In our localization algorithm, each stationary sensor node needs less consumption of energy for receiving the message. So our localization algorithm needs less overhead than the other two schemes for

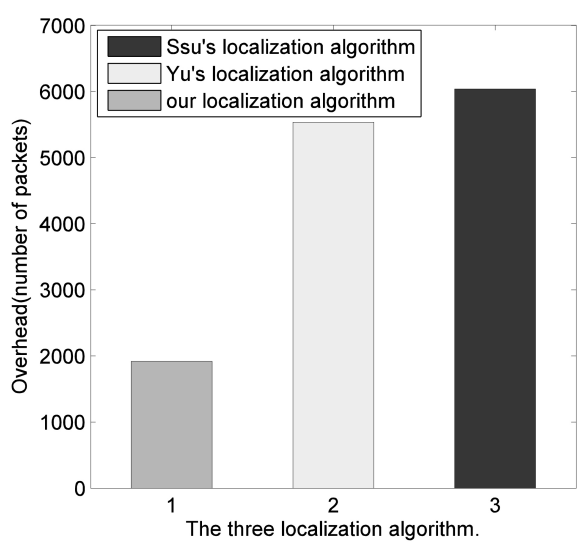

Fig. 15. Localization overhead 
localization.

2. Average localization error: It can be defined as the average difference between estimated locations (Xei, Yei) and actual locations (Xi, Yi) of all sensor nodes [4].

$$
\Delta E_{a v}=\frac{\sum_{i=1}^{N} \sqrt{(X e i-X i)^{2}+(Y e i-Y i)^{2}}}{N}
$$

Our algorithm average localization errors and other schemes localization errors are shown in Fig.16.

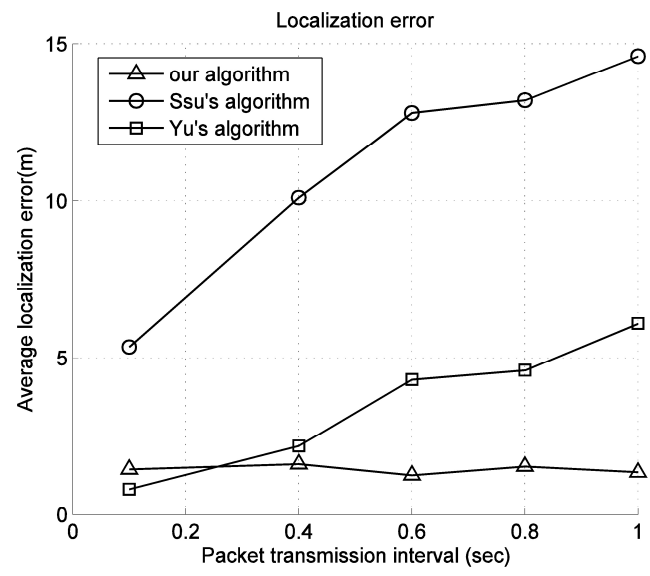

Fig. 16. Localization error

\subsection{Simulation Results}

In our simulations, three typical localization schemes, Yu'algorithm [2], Ssu'salgorithm [4] were evaluated for performance comparison.

1. Average overhead: The average overhead for the three algorithms was simulated and results are shown in Fig.15. We can clearly see that our localization scheme outperforms other schemes. In our localization algorithm, each stationary sensor node needs less overhead to locate.

2. Average Localization Errors: In all three localization algorithms, sensor locations were computed based on beacon points that are selected by receiving packets from mobile beacon nodes. The localization error of our algorithm is less than the other algorithms and our algorithm is less affected by packet transmission interval.

\section{RESULtS}

We have presented an angle energy efficient localization algorithm by using a mobile beacon node that is equipped with a GPS receiver and RF transmitter. It is based on the estimated distance between the beacon node and stationary sensor node using RF. The mobile beacon node 
makes use of Random Direction Mobility Model and moves over the sensor area and transmits its location information. Each stationary sensor node uses the information to compute its location, which is performed locally. Our proposed localization scheme was evaluated using OPNET 8.1 and compared with Ssu's and Yu's localization schemes. The results show that our localization scheme outperforms the other two schemes in terms of energy efficiency (overhead) and accuracy.

\section{REFERENCES}

[1] Baoli Zhang and Fengqi Yu, "An Energy Efficient Localization Algorithm for Wireless Sensor Networks Using a Mobile Anchor Node", International Conference on Information and Automation, June, 2008, pp.215-216.

[2] G.Yu, F.Yu and L. Feng, "A Three Dimensional Localization Algorithm Using a Mobile Anchor Node under Wireless Channel”, International Joint Conference on Neural Networks(IJCNN), 2008, pp.477-483.

[3] T.V Srinath, "Localization in Resource Constrained Sensor Networks Using A Mobile Beacon with In-ranging" Wireless and Optical Communications Networks, 2006 IFIP International Conference, April, 2006, pp.5.

[4] K.-F. Ssu, C.-H. Ou and H. C. Jiau, "Localization with mobile anchor points in wireless sensor networks," IEEE Trans. Vehicular Technology. Taiwan, Vol.54, No.3, May, 2005, pp.1187-1197.

[5] Nissanka B.Priyantha, Anit Chakraborty, and Hari Balakrishnan, "The Cricket Location-Support System", Proceedings of 6th Annual ACM/IEEE International Conference on Mobile Computing and Networking (MobiCom 2000), August, 2000, pp.32-43.

[6] J. Hightower,G. Boriello, and R.Want, "SpotON: An indoor 3D location sensing technology based on RF signal strength," Univ. of Washington, Tech. Rep. UW CSE 00-02-02, Feb., 2000.

[7] P. Bahl and V. N. Padmanabhan, "RADAR: an in-building RF-based userlocation and tracking system," in Proc. IEEE Joint Conf. IEEE Computer Communications Societies (INFOCOM), Tel Aviv, Israel, Mar., 2000, pp.775-784.

[8] P. Bergamo and G. Mazzini, "Localization in sensor networks with fading and mobility," in Proc. IEEE Int. Symp. Personal, Indoor Mobile Radio Communications (PIMRC), Lisbon, Portugal, Sep., 2002, pp.750-754.

[9] L. Cong and W. Zhuang, "Hybrid TDOA/AOA mobile user location for wideband CDMA cellular systems," IEEE Trans. Wireless Commun., Vol.1, Jul., 2002, pp.439-447.

[10] M. McGuire, K. N. Plataniotis, and A. N. Venetsanopoulos, "Location of mobile terminals using time measurements and survey points," IEEE Trans. Veh. Technol., Vol.52, Jul., 2003 , pp.999-1011.

[11] N. Bulusu, J. Heidemann, and D. Estrin, "GPS-less low cost outdoorlocalization for very small devices", IEEE Personal Commun., Vol.7, Oct., 2000, pp.28-34.

[12] B. Liang and Z. Haas. Predictive distance-based mobility management for PCS networks. In Proceedings of the Joint Conference of the IEEE Computer and Communications Societies (INFOCOM), March, 1999.

[13] D. Johnson and D. Maltz. Dynamic source routing in ad hoc wireless networks. In T. Imelinsky and H.Korth, editors, Mobile Computing, pages 153-181. Kluwer Academic Publishers, 1996.

[14] X. Hong, M. Gerla, G. Pei, and C. Chiang. A group mobility model for ad hoc wireless networks. In Proceedings of the ACM International Workshop on Modeling and Simulation of Wireless and Mobile Systems (MSWiM), August, 1999.

[15] E. Royer, P.M. Melliar-Smith, and L. Moser. An analysis of the optimum node density for ad hoc mobile networks. In Proceedings of the IEEE International Conference on Communications (ICC), 2001.

[16] CHEN Juan, LI Chang-geng, NING Xin-xian. Node Localization of Wireless Sensor Networks Based on Mobile Beacon.[J]. Chinese Journal of Sensors and Actuators. 2009, 22(1): 123-125.(in Chinese). 
[17] M.L. Sichitiu and V. Ramadurai, "Localization of wireless sensor networks with a mobile beacon", Center for Advances Computing Communications, North Carolina State Univ. Tech. Rep. TR-03/06. Jul., 2003.

[18] G.L. Sun and W. Guo, "Comparison of distributed localization algorithms for sensor network with a mobile beacon", in Proc. IEEE Int. Conf. Networking, Sensing Control (ICNSC), Taipei, Taiwan, R.O.C. Mar., 2004, pp.36-540.

[19] Estrin D.Wireless Sensor Networks, Tutorial Part IV:Sensor Network Protocols.In Proceedings of The Eighth Annual International Conference on Mobile Computing and Networking(MOBICOM),2002.

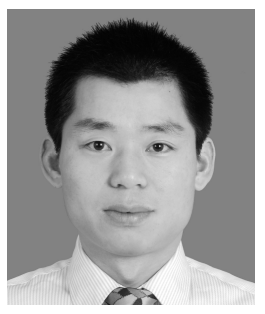

\section{Kezhong Liu}

He received a B.S. and M.S. degrees in traffic engineering from Wuhan University of Technology, Wuhan, China, in 1998 and 2001, respectively, and a Ph.D. degree in communication engineering from Huazhong University of Technology, Wuhan, China, in 2006.

$\mathrm{He}$ is an Associate Professor in the Navigation School of Wuhan University of Technology. His current research interests include distributed localization and information processing in wireless sensor networks, and distributed data fusion for target tracking and marine surveillance networks.

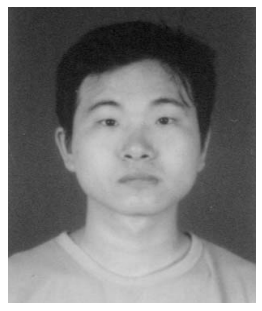

\section{Ji Xiong}

he He is a master degree student in the department of Traffic Information Engineering and Control of Wuhan University of Technology, Wuhan, China. From September 2008 to December 2010, he studied for, and received a B.S. degree in Wuhan University of Technology in 2006. His research interest in Localization of WSN. 